\title{
Intracerebral hemorrhage in COVID-19 patients with pulmonary failure - a propensity score matched registry study
}

\section{Corinna N. Lang ( $\square$ corinna.nadine.lang@uniklinik-freiburg.de )}

Heart Center Freiburg University, Department of Cardiology and Angiology I, Faculty of Medicine, University of Freiburg, Freiburg, Germany

\section{Johanna S. Dettinger}

Heart Center Freiburg University, Department of Cardiology and Angiology I, Faculty of Medicine, University of Freiburg, Freiburg, Germany

\section{Michael Berchtold-Herz}

Heart Center Freiburg University, Department of Cardiovascular Surgery, Faculty of Medicine, University of Freiburg, Freiburg, Germany

\section{Stefan Utzolino}

Department of General and Visceral Surgery, Medical Center, University of Freiburg, Freiburg, Germany

\section{Xavier Bemtgen}

Heart Center Freiburg University, Department of Cardiology and Angiology I, Faculty of Medicine, University of Freiburg, Freiburg, Germany

\section{Viviane Zotzmann}

Heart Center Freiburg University, Department of Cardiology and Angiology I, Faculty of Medicine, University of Freiburg, Freiburg, Germany

\section{Bonaventura Schmid}

Department of Emergency Medicine, Faculty of Medicine, University of Freiburg, Freiburg, Germany

\section{Paul M. Biever}

Heart Center Freiburg University, Department of Cardiology and Angiology I, Faculty of Medicine, University of Freiburg, Freiburg, Germany

\section{Christoph Bode}

Heart Center Freiburg University, Department of Cardiology and Angiology I, Faculty of Medicine, University of Freiburg, Freiburg, Germany

\section{Katharina Müller-Peltzer}

Department of Radiology, Faculty of Medicine, University of Freiburg, Freiburg, Germany

\section{Daniel Duerschmied}

Heart Center Freiburg University, Department of Cardiology and Angiology I, Faculty of Medicine, University of Freiburg, Freiburg, Germany

\section{Tobias Wengenmayer}


Heart Center Freiburg University, Department of Cardiology and Angiology I, Faculty of Medicine, University of Freiburg, Freiburg, Germany

\section{Wolf-Dirk Niesen}

Department of Neurology, Faculty of Medicine, University of Freiburg, Freiburg, Germany

\section{Dawid L. Staudacher}

Heart Center Freiburg University, Department of Cardiology and Angiology I, Faculty of Medicine, University of Freiburg, Freiburg, Germany

\section{Research Article}

Keywords: intracerebral hemorrhage, ARDS, COVID-19

Posted Date: August 11th, 2020

DOI: https://doi.org/10.21203/rs.3.rs-56258/v1

License: (c) (1) This work is licensed under a Creative Commons Attribution 4.0 International License. Read Full License

Version of Record: A version of this preprint was published at Neurocritical Care on February 23rd, 2021. See the published version at https://doi.org/10.1007/s12028-021-01202-7. 


\section{Abstract}

Background: Hypercoagulopathy in coronavirus disease 2019 (COVID-19) causing deep vein thrombosis and pulmonary artery embolism necessitate systemic anticoagulation. Case reports of intracerebral hemorrhages in ventilated COVID-19 patients warrant precaution. It is unclear however, if COVID-19 patients with acute respiratory distress syndrome (ARDS) with and without extracorporeal membrane oxygenation therapy $(\mathrm{ECMO})$ have more intracerebral hemorrhages $(\mathrm{ICH})$ compared to other ARDS patients.

Methods: We conducted a retrospective observational single center study enrolling all patients with ARDS from 01/2018-05/2020. Patients with ARDS positive for SARS-CoV2 PCR were allocated to the COVID-19 group. Propensity score matching was performed for age, ECMO and risk of bleeding according to HASBLED score.

Results: A total of 163 , mostly severe ARDS patients were identified, 116 (71.2\%) without COVID-19 and $47(28.8 \%)$ positive for SARS-CoV-2. The two groups were comparable concerning the main confounders of ICH including age, HAS-BLED score, need for ECMO-therapy as well as anticoagulation levels reported. In $63 / 163$ cases (38.7\%), veno-venous ECMO therapy was required and ICU survival was $52.8 \%$. Although HAS-BLED-score on admission was generally low (1.6 \pm 1.3$)$, intracerebral hemorrhage was detected in 22 patients $(13.5 \%)$ with no statistical difference between the groups (11.2 vs. $19.1 \%$ with and without SARS$\mathrm{CoV}-2$, respectively, $\mathrm{p}=0.21)$. Propensity score matching confirmed similar intracerebral bleeding rates in both groups ( 12.8 vs. $19.1 \%$ with and without SARS-CoV-2, respectively, $p=0.57$ ).

Conclusions: Intracerebral hemorrhage was detectable in every tenth patient with ARDS. We found no statistically significant increased bleeding rate in patients with ARDS due to COVID-19 compared to other causes of ARDS.

\section{Introduction}

Several studies point out a state of hypercoagulability occurring in coronavirus disease 2019 (COVID-19) probably due to inflammatory changes comparable to disseminated intravascular coagulopathy. $(1,2)$ Additionally, clinical and pathohistological reports about micro- and macrothrombosis as typical complications in critically ill COVID-19 patients emphasize the need for adjusted thromboprophylaxis treatment.(3-5) Until today, there is no concrete evidence to manage thromboprophylaxis beyond standard indications. $(6,7)$ Ongoing studies focus on more aggressive anticoagulation in order to avoid thromboembolic complications (clinicaltrials.gov last accessed 06/17/2020; 23 trials prophylactic, intermediate, therapeutic heparin doses). Nevertheless, higher anticoagulation targets are already proposed by some clinicians.(8)

Whereas pulmonary embolism, and deep vein thrombosis have been documented frequently in hospitalized COVID-19 patients (3), major bleedings have not been reported on a large scale in these 
cohorts even though there seems to be a higher event rate of cerebral events.(9) By now, three cases have been reported with massive intracerebral hemorrhage. $(10,11)$

During the treatment of our cohort of critical ill COVID-19 patients, three fatal intracerebral hemorrhages occurred. We therefore raised the question if intracerebral hemorrhage is more common in critically ill COVID-19 patients with respiratory failure compared to the general population of patients with acute respiratory distress syndrome (ARDS).

\section{Methods}

\section{Patient selection}

Consecutively, patients with ARDS (ICD-10 code J80.01; J80.02; J80.03; J80.03; J80.09) were extracted from the hospital data system from 01/01/2018-05/31/2020 and followed until 06/13/2020 and included in our retrospective observational study performed at the University hospital of Freiburg, Germany. For the COVID-19 group critically ill patients with PCR-confirmed SARS-CoV-2 infection and severe respiratory failure were enrolled from 03/2020-05/2020 (first admission 03/08/2020) and followed until 06/13/2020 (COVID-19). The study protocol was approved by the local ethics committee (Ethik-Kommission der Albert-Ludwigs-Universität Freiburg im Breisgau, file number 333/20).

Data collection and statistics

Clinical data reported in this study was collected from our hospital data system and from documentation of transferring hospitals and included: age, sex, sepsis-related organ failure assessment score (SOFA), length of stay, intensive care unit (ICU) survival, invasive mechanical ventilation (IMV) and extracorporeal therapies (renal replacement therapy, RRT and extracorporeal membrane oxygenation, ECMO). ARDS was classified according to the Berlin classification.(12) If ECMO was necessary, ARDS was considered "severe", since calculation of the Horovitz index required for the Berlin classification is not valid in ECMO. To evaluate the overall bleeding risk, we assessed the HAS-BLED score (a well validated score for bleeding risk in chronic atrial fibrillation as well as in acute disease) on admission and 48 hours prior to bleeding. $(13,14)$ Values of non-COVID-19 patients and COVID-19 patients were compared with chi-square test and t-test. Propensity score matching (1:1) was performed between the two groups matched for age, ECMO and the HAS-BLED score using SPSS (version 26, IBM, NYC, USA) and the optimal matching algorithm with a caliper of 0.1 . Results were considered as statistically significant when $p$-value was below 0.05. Graphs were made using Prism, version 8 (GraphPad, San Diego, USA).

\section{Patient management}

Patients were transferred from the emergency department, from internal wards or from primary and secondary treatment centers. Our center is a tertiary treatment center with a high percentage of hospital transferals for ECMO evaluation or after ECMO pick up. All patients received guideline-conform ARDS treatment confirmed in house-intern standard operating procedures for lung-protective ventilation, 
therapeutic positioning maneuvers as proning and further supportive therapies according to the underlying cause of the disease. $(7,15)$ Anticoagulation strategies followed house-intern standard operating procedures and current guidelines. Since 04/03/2020 COVID-19 patients on ECMO were managed with a coagulation target of partial thromboplastin time (aPTT) at 50-70s. In case of thrombotic events under unfractionated heparin a timely switch to argatroban was performed in these patients; unfractionated heparin being the standard medication for thromboprophylaxis and therapeutic anticoagulation. Non-COVID-19 ECMO patients were managed with a coagulation target of aPTT at 4050 s.

Intracerebral hemorrhage

Cerebral imaging was indicated according to clinical judgment and enlisted for analysis if dated during ICU therapy or shortly after. An experienced radiologist and neurologist re-read all cerebral computed tomography (CCT) scans and cerebral magnet tomography scans image-by-image according to occurrence of intracerebral hemorrhage and pathogenesis. Additionally, new ischemic events were detected and classified according to their pathogenesis; preexisting microangiopathy according the Fazekas classification was looked at.(16) The evaluation of the Fazekas classification was not performed in case of massive cerebral edema or massive intracerebral bleeding.

\section{Results}

Baseline characteristics

From January 2018 to May 2020, 163 ARDS patients were identified and included into the analysis. The 116 non-COVID-19 patients presented with ARDS due to an infection in 70\%; other etiologies are given in the electronic supplemental material (ESM) table S4. Since March 2020, we included 47 critically ill COVID-19 patients with intensive care therapy due to pulmonary failure (Table 1, Figure 1). On average, patients were aged $60 \pm 15$ (24-92) years. There were more female patients in the non-COVID-19 group $(42.4 \%$ vs. $19.1 \%$ in non-COVID-19 vs. COVID- 19 , respectively $p=0.005)$ and the SOFA score was higher (11 \pm 4 vs $9 \pm 4$ in non-COVID-19 vs. COVID-19, respectively, $p=0.006)$.

A total of 63/163 (38.7\%) patients underwent ECMO therapy without difference between the groups. Furthermore, length of ICU stay, days on IMV and days on ECMO did not differ between the two groups. ICU survival was $52.8 \%$ and not different between the groups ( $52.6 \%$ vs. $53.2 \%)$, mode of death is given in the ESM (Table S3).

Intracerebral hemorrhage

Out of 163 patients, 96 (58.3\%) had a cerebral scan (Table 3). The rate of cerebral imaging did no differ between the two groups (60.3\% vs 55.3\%; non-COVID-19 vs COVID-19). In the non-COVID-19 group, we detected 13 intracerebral hemorrhages compared to 9 in the COVID-19 group (see figure 2). No significant 
difference was found between the two groups according to the occurrence of any intracerebral hemorrhage or a fatal intracerebral hemorrhage (Figure 3, ESM Table S3).

The rate of atypical bleedings was numerically higher in the non-COVID-19 group (7/13 vs. 2/9, $p=0.203)$ not reaching statistical significance. Multiple intracerebral hemorrhage however were only detected in the COVID-19 group (0/13 vs. 4/9, $p=0.017)$. After propensity score matching, results could be confirmed with similar rates of intracerebral hemorrhage in patients without and with COVID-19 (Figure 3). No difference was detectable in new strokes (12.9\% vs. $6.4 \%$ for non-COVID-19 and COVID-19 group, respectively) with a high rate of embolic origin (77.8\%) and no proximal vessel occlusions.

Risk factors for bleeding and anticoagulation

On admission, patients presented with a low HAS-BLED score 1.6 $\pm 1.3(0-4)$ similar between the two groups (Table 2). The HAS-BLED score 48 hours prior to intracerebral hemorrhage was similar in nonCOVID-19 and COVID-19 patients. Alcohol abuse (a risk factor in the HAS-BLED score) was detected significantly more often in the non-COVID-19 patients $(p=0.007)$.

Anticoagulation targets for individual patients did not differ during the ICU stay with a higher tendency to reach a therapeutic aPTT in COVID-19 patients (33.6\% vs. 44.7\%; non-COVID-19 vs COVID-19). Laboratory tests were similar between the groups concerning platelet count, INR and aPTT (Table S2).

Unfractionated heparin was used significantly more often in non-COVID-19 patients $(p=0.01)$; whereas more COVID-19 patients were switched to argatroban $(\mathrm{p}<0.001)$. Blood pressure excess or aPTT excess 48 hours prior to the intracerebral hemorrhage were rare. The Fazekas score between the groups was similar ( $1.3 \pm 0.9$ vs $1.0 \pm 0.8$; non-COVID-19 vs COVID-19, $p=0.293)$.

\section{Discussion}

Intracerebral hemorrhages and ARDS

We found an intracerebral hemorrhage in $13.5 \%$ of all ARDS patients with a tendency towards higher bleeding rate in COVID-19 patients not reaching statistical significance neither in the whole cohort nor after propensity score matching. To our best knowledge, this is the first study elucidating the risk of intracerebral hemorrhages in COVID-19. So far, only case reports of devastating intracerebral hemorrhages have been published. $(10,11)$

We can only speculate why the rate of intracerebral hemorrhages is so high in the ARDS collective. For SARS-CoV2, neuroinvasion and neurotropism have been reported and the Coronavirus was isolated from cerebrospinal fluid and brain tissue. $(17,18)$ In a systematic review, long-term cognitive impairment in a collective of mixed ARDS patients was detected in 70-100\% patients at hospital discharge and in $20 \% 5$ years after the ARDS.(19) An imaging study revealed cerebral and hippocampal atrophy one year after ARDS compared to healthy controls.(20) Since the rate of intracerebral hemorrhage in our study detected in COVID-19 seems comparable to other ARDS, a more general pathomechanism with cerebral damage 
due to systemic inflammation might be responsible. Due to the retrospective nature of our study, we cannot determine if intracerebral hemorrhage in ARDS is caused by an embolic event with secondary hemorrhage or if bleedings are caused primarily by neurotropism and local endothelitis.

The observed rate of intracerebral hemorrhages during veno-venous ECMO $(20.1 \% ; 13 / 63)$ therapy matches the rate reported by Fletcher-Sandersjöö et al. 20\% (65/351 patients) investigating intracranial hemorrhages during mixed adult ECMO therapy. A systematic literature review suggested incidence of intracranial hemorrhage between $1.8-21 \%$ during veno-venous or veno-arterial ECMO. $(21,22)$ ICU survival in our cohort (52.8\%) is lower than reported by other data for severe ARDS (38\%).(23) Our registry contains severely ill patients as suggested by the predicted mortality of $50.0 \%$ according to the SOFA score (24), which might explain these differences.

Anticoagulation during ARDS

In our registry, $60.1 \%$ of all ARDS patients were on anticoagulation other than for prophylaxis of deep vein thrombosis. This might partly explain the high incidence of intracerebral bleeding reported. On the other hand, some phenotypes of pulmonary failure proceed with a hyperinflammatory immune answer, circulatory and subsequent multi-organ failure. The underlying coagulopathy results in clinical complications such as deep vein thrombosis, pulmonary artery thrombosis or clotting on extracorporeal organ replacement therapies. Laboratory measures result in elevated d-dimers, prolonged prothrombin time and thrombocytopenia along with elevated markers for inflammation (c-reactive protein, interleucin 6, ferritin).(25-27) A local reaction such as a virus associated endothelitis or a systemic inflammation might also trigger these prothrombotic events. $(2,28)$

\section{Limitations:}

We have to point out several limitations of our study. First, the results of our study are limited in consideration of the small patient numbers. Second, as tertiary treatment center our patients were selected with moderate and severe ARDS as well as high SOFA scores. Results might be different in first and secondary treatment centers. Third, CCT scans were indicated by clinical judgement. It remains unclear how many clinically silent events did occur. Fourth, our anticoagulation regimes under ECMO therapy are empiric for COVID-19 and non-COVID-19 patients and tended to be more aggressive for COVID-19 patients. Fifth, more male patients were in the non-COVID-19 group with male gender being a known factor for intracerebral hemorrhage.(29) In our cohort, we did detected similar bleeding rates in females and males with a tendency towards more bleeding in female patients $(19.0 \%$ vs $10.5 \%$ for females and males, respectively, $\mathrm{p}=0.153)$. Finally, the rate of intracerebral hemorrhage in our cohort with $13.5 \%(22 / 163)$ and the rate for strokes of $11.0 \%$ (18/163) might not be representative for cohorts in primary and secondary treatment centers with lower SOFA scores and lower rates of organ replacement therapies.

\section{Conclusions}


Intracerebral hemorrhage was detectable in every tenth patient with ARDS. We found no statistical significant increase in bleeding rate in patients with ARDS due to COVID-19 compared to other causes of ARDS. These data should prompt future trials.

\section{Abbreviations}

aPTT, partial thromboplastin time

ARDS, severe acute respiratory distress syndrome

BMl, body mass index

$\mathrm{cCT}$, cerebral computed tomography

COVID-19, coronavirus disease 2019

ESM, electronic supplemental material

ER, emergency room

ECMO, extracorporeal membrane oxygenation

ICU, intensive care unit

$\mathrm{ICH}$, intracerebral hemorrhage

IMV, invasive mechanical ventilation

HAS-BLED Score, validated score for bleeding risk in chronic atrial fibrillation as well as in acute disease

RRT, renal replacement therapy

SOFA Score, sepsis-related organ failure assessment score

SARS-CoV-2, severe acute respiratory coronavirus 2

PEEP, positive end-expiratory pressure

\section{Declarations}

Ethics approval and consent to participate: The local ethics committee approved the study protocol (Ethik-Kommission der Albert-Ludwigs-Universität Freiburg im Breisgau, file number 333/20).

Consent for publication: According to the local ethics committee according to the retrospective character of the study written or verbal consent was not necessary. 
Availability of data and materials: The datasets during and/or analyzed during the current study available from the corresponding author on reasonable request.

Conflict of interests: The authors do hereby declare no conflict of interests.

Permissions information: The authors do hereby declare that all illustrations and figures in the manuscript are entirely original and do not require reprint permission.

\section{Funding: None.}

Authors' contributions: CNL concepted and designed the study, performed data acquisition, analysis and interpretation of the data, drafted and revised the manuscript. JSD performed data acquisition and interpretation of the data, revised the manuscript. $\mathrm{MBH}$ performed interpretation of the data, revised the manuscript. SU performed interpretation of the data, revised the manuscript. XB performed data acquisition and interpretation of the data, revised the manuscript. VZ performed interpretation of the data, revised the manuscript. BS performed interpretation of the data, revised the manuscript. PMB performed interpretation of the data, revised the manuscript. CB performed interpretation of the data, revised the manuscript. KMP performed interpretation of the data, performed interpretation of the CCT scans, revised the manuscript. DD performed interpretation of the data, revised the manuscript. TW performed interpretation of the data, revised the manuscript. WDN concepted and designed the study, performed interpretation of the CCT scans and data, revised the manuscript. DLS concepted and designed the study, created the artwork, performed data analysis and revised the manuscript. All authors read and approved the final manuscript.

Acknowledgements: We thank Michaela Kirchenmayer for the collection of extern documentation.

\section{References}

1. Mucha SR, Dugar S, McCrae K, Joseph DE, Bartholomew J, Sacha G, u. a. Coagulopathy in COVID-19. Cleve Clin J Med. 14. Mai 2020;

2. Henry BM, Vikse J, Benoit S, Favaloro EJ, Lippi G. Hyperinflammation and derangement of reninangiotensin-aldosterone system in COVID-19: A novel hypothesis for clinically suspected hypercoagulopathy and microvascular immunothrombosis. Clin Chim Acta Int $\mathrm{J}$ Clin Chem. August 2020;507:167-73.

3. Klok FA, Kruip MJHA, van der Meer NJM, Arbous MS, Gommers D, Kant KM, u. a. Confirmation of the high cumulative incidence of thrombotic complications in critically ill ICU patients with COVID-19: An updated analysis. Thromb Res. 30. April 2020;

4. Klok FA, Kruip MJHA, van der Meer NJM, Arbous MS, Gommers DAMPJ, Kant KM, u. a. Incidence of thrombotic complications in critically ill ICU patients with COVID-19. Thromb Res [Internet]. 10. April 
2020 [zitiert 29. Mai 2020]; Verfügbar unter:

https://www.ncbi.nlm.nih.gov/pmc/articles/PMC7146714/

5. Wichmann D, Sperhake J-P, Lütgehetmann M, Steurer S, Edler C, Heinemann A, u. a. Autopsy Findings and Venous Thromboembolism in Patients With COVID-19: A Prospective Cohort Study. Ann Intern Med. 6. Mai 2020;

6. Thachil J, Tang N, Gando S, Falanga A, Cattaneo M, Levi M, u. a. ISTH interim guidance on recognition and management of coagulopathy in COVID-19. J Thromb Haemost JTH. 2020;18(5):1023-6.

7. Alhazzani W, Møller MH, Arabi YM, Loeb M, Gong MN, Fan E, u. a. Surviving Sepsis Campaign: guidelines on the management of critically ill adults with Coronavirus Disease 2019 (COVID-19). Intensive Care Med. 2020;46(5):854-87.

8. Barrett CD, Moore HB, Yaffe MB, Moore EE. ISTH interim guidance on recognition and management of coagulopathy in COVID-19: A Comment. J Thromb Haemost JTH. 17. April 2020;

9. Merkler AE, Parikh NS, Mir S, Gupta A, Kamel H, Lin E, u. a. Risk of Ischemic Stroke in Patients with Covid-19 versus Patients with Influenza. medRxiv. 21. Mai 2020;2020.05.18.20105494.

10. Sharifi-Razavi A, Karimi N, Rouhani N. COVID-19 and intracerebral haemorrhage: causative or coincidental? New Microbes New Infect. Mai 2020;35:100669.

11. Carroll E, Lewis A. Catastrophic Intracranial Hemorrhage in Two Critically III Patients with COVID-19. Neurocrit Care. 26. Mai 2020;1-5.

12. ARDS Definition Task Force, Ranieri VM, Rubenfeld GD, Thompson BT, Ferguson ND, Caldwell E, u. a. Acute respiratory distress syndrome: the Berlin Definition. JAMA. 20. Juni 2012;307(23):2526-33.

13. Klok FA, Niemann C, Dellas C, Hasenfuß G, Konstantinides S, Lankeit M. Performance of five different bleeding-prediction scores in patients with acute pulmonary embolism. J Thromb Thrombolysis. Februar 2016;41(2):312-20.

14. Zhu W, He W, Guo L, Wang X, Hong K. The HAS-BLED Score for Predicting Major Bleeding Risk in Anticoagulated Patients With Atrial Fibrillation: A Systematic Review and Meta-analysis. Clin Cardiol. September 2015;38(9):555-61.

15. Griffiths MJD, McAuley DF, Perkins GD, Barrett N, Blackwood B, Boyle A, u. a. Guidelines on the management of acute respiratory distress syndrome. BMJ Open Respir Res. 1. Mai 2019;6(1):e000420.

16. Fazekas F, Kleinert R, Roob G, Kleinert G, Kapeller P, Schmidt R, u. a. Histopathologic analysis of foci of signal loss on gradient-echo T2*-weighted MR images in patients with spontaneous intracerebral hemorrhage: evidence of microangiopathy-related microbleeds. AJNR Am J Neuroradiol. April 1999;20(4):637-42.

17. Asadi-Pooya AA, Simani L. Central nervous system manifestations of COVID-19: A systematic review. J Neurol Sci. 15. Juni 2020;413:116832.

18. Mao L, Jin H, Wang M, Hu Y, Chen S, He Q, u. a. Neurologic Manifestations of Hospitalized Patients With Coronavirus Disease 2019 in Wuhan, China. JAMA Neurol. Juni 2020;77(6):1-9. 
19. Sasannejad C, Ely EW, Lahiri S. Long-term cognitive impairment after acute respiratory distress syndrome: a review of clinical impact and pathophysiological mechanisms. Crit Care [Internet]. 12. November 2019 [zitiert 18. Juni 2020];23. Verfügbar unter: https://www.ncbi.nlm.nih.gov/pmc/articles/PMC6852966/

20. Hopkins RO, Gale SD, Weaver LK. Brain atrophy and cognitive impairment in survivors of Acute Respiratory Distress Syndrome. Brain Inj. März 2006;20(3):263-71.

21. Fletcher-Sandersjöö A, Thelin EP, Bartek J, Elmi-Terander A, Broman M, Bellander B-M. Management of intracranial hemorrhage in adult patients on extracorporeal membrane oxygenation (ECMO): An observational cohort study. PLoS ONE [Internet]. 21. Dezember 2017 [zitiert 18. Juni 2020];12(12). Verfügbar unter: https://www.ncbi.nlm.nih.gov/pmc/articles/PMC5739492/

22. Fletcher-Sandersjöö A, Thelin EP, Bartek J, Broman M, Sallisalmi M, Elmi-Terander A, u. a. Incidence, Outcome, and Predictors of Intracranial Hemorrhage in Adult Patients on Extracorporeal Membrane Oxygenation: A Systematic and Narrative Review. Front Neurol [Internet]. 6. Juli 2018 [zitiert 18. Juni 2020];9. Verfügbar unter: https://www.ncbi.nlm.nih.gov/pmc/articles/PMC6043665/

23. Máca J, Jor O, Holub M, Sklienka P, Burša F, Burda M, u. a. Past and Present ARDS Mortality Rates: A Systematic Review. Respir Care. Januar 2017;62(1):113-22.

24. Vincent JL, Moreno R, Takala J, Willatts S, De Mendonça A, Bruining H, u. a. The SOFA (Sepsisrelated Organ Failure Assessment) score to describe organ dysfunction/failure. On behalf of the Working Group on Sepsis-Related Problems of the European Society of Intensive Care Medicine. Intensive Care Med. Juli 1996;22(7):707-10.

25. Lippi G, Favaloro EJ. D-dimer is Associated with Severity of Coronavirus Disease 2019: A Pooled Analysis. Thromb Haemost. Mai 2020;120(5):876-8.

26. Lippi G, Plebani M, Henry BM. Thrombocytopenia is associated with severe coronavirus disease 2019 (COVID-19) infections: A meta-analysis. Clin Chim Acta Int J Clin Chem. 13. März 2020;506:145-8.

27. Henry BM, de Oliveira MHS, Benoit S, Plebani M, Lippi G. Hematologic, biochemical and immune biomarker abnormalities associated with severe illness and mortality in coronavirus disease 2019 (COVID-19): a meta-analysis. Clin Chem Lab Med. 10. April 2020;

28. Varga Z, Flammer AJ, Steiger P, Haberecker M, Andermatt R, Zinkernagel AS, u. a. Endothelial cell infection and endotheliitis in COVID-19. Lancet Lond Engl. 02 2020;395(10234):1417-8.

29. An SJ, Kim TJ, Yoon B-W. Epidemiology, Risk Factors, and Clinical Features of Intracerebral Hemorrhage: An Update. J Stroke. Januar 2017;19(1):3-10.

\section{Tables}

Table 1: baseline characteristics of non-COVID-19 patients and COVID-19 patients 


\begin{tabular}{|c|c|c|c|}
\hline & non-COVID-19 & COVID-19 & all patients \\
\hline Number of patients & $116(71.2 \%)$ & $47(28.8 \%)$ & $163(100 \%)$ \\
\hline Age [years] & $58 \pm 15(24-83)$ & $66 \pm 13(31-92)$ & $60 \pm 15(24-92)$ \\
\hline Female gender & $49(42.2 \%)$ & 9 (19.1\%) & $58(35.6 \%)$ \\
\hline Body mass index [kg/m几] & $30 \pm 11(16-83)$ & $28 \pm 6(18-51)$ & $30 \pm 10(16-83)$ \\
\hline Adipositas $[\mathrm{BMI} \geq 30 \mathrm{~kg} / \mathrm{m}[]$ & $32(33.0 \%)$ & $13(28.9 \%)$ & $45(31.5 \%)$ \\
\hline Length of stay [days] & $18 \pm 16(1-76)$ & $23 \pm 20(1-89)$ & $19 \pm 17(1-89)$ \\
\hline SOFA Score & $11 \pm 4(2-19)$ & $9 \pm 4(2-17)$ & $10 \pm 4(2-19)$ \\
\hline ICU survival & $61(52.6 \%)$ & $25(53.2 \%)$ & $86(52.8 \%)$ \\
\hline \multicolumn{4}{|l|}{ ARDS } \\
\hline Mild & 0 & 0 & 0 \\
\hline Moderate & $35(30.2 \%)$ & $18(38.3 \%)$ & $53(32.5 \%)$ \\
\hline Severe & $81(69.8 \%)$ & $29(61.7 \%)$ & $110(67.5 \%)$ \\
\hline $\mathrm{paO}_{2} / \mathrm{FiO}_{2}$ (on day 1 ) & $110 \pm 39(36-222)$ & $113 \pm 38(35-227)$ & $111 \pm 38(35-227)$ \\
\hline Highest PEEP (on day 1) & $13 \pm 4(5-20)$ & $12 \pm 4(5-19)$ & $12 \pm 4(5-20)$ \\
\hline Representative $\mathrm{FiO}_{2}$ at highest PEEP (on day 1) & $58 \pm 17(30-100)$ & $65 \pm 19(40-100)$ & $60 \pm 18(30-100)$ \\
\hline Invasive mechanical ventilation (IMV) & $106(91.4 \%)$ & $40(85.1 \%)$ & $146(89.6 \%)$ \\
\hline Duration of IMV [days] & $19 \pm 20(1-129)$ & $23 \pm 22(1-89)$ & $20 \pm 21(1-129)$ \\
\hline Renal replacement therapy (RRT) & $29(25.0 \%)$ & $13(27.7 \%)$ & $42(25.8 \%)$ \\
\hline Extracorporeal membrane oxygenation (ECMO) & $49(42.2 \%)$ & $14(29.8 \%)$ & $63(38.7 \%)$ \\
\hline Duration of ECMO [days] & $16 \pm 17(2-72)$ & $22 \pm 20(2-71)$ & $17 \pm 18(2-72)$ \\
\hline
\end{tabular}

Baseline characteristics are displayed for all patients, in non-COVID-19-ARDS and patients with COVID-19ARDS. Data are $\mathrm{n}(\%)$ or mean with standard deviation and range. If values differ significantly between non-COVID and COVID-patients, they are marked bold: female: $p=0.005$; SOFA score: $p=0.006$; representative $\mathrm{FiO}_{2}: \mathrm{p}=0.023$.

Table 2: risk factors for bleeding 


\begin{tabular}{|c|c|c|c|}
\hline & non-COVID-19 & COVID-19 & all patients \\
\hline Number of patients & $116(71.2 \%)$ & 47 (28.8\%) & $163(100 \%)$ \\
\hline HAS-BLED score (on admission) & $1.5 \pm 1.3(0-4)$ & $1.7 \pm 1.3(0-4)$ & $1.6 \pm 1.3(0-4)$ \\
\hline Hypertension & $48(41.4 \%)$ & $26(55.3 \%)$ & $74(45.4 \%)$ \\
\hline Abnormal liver function & 15 (15.5\%) & 0 & $15(9.2 \%)$ \\
\hline Abnormal kidney function & $18(15.5 \%)$ & $9(19.1 \%)$ & $27(16.6 \%)$ \\
\hline Former stroke & $1(0.9 \%)$ & $2(4.3 \%)$ & $3(1.8 \%)$ \\
\hline Former tendency to bleed & $2(1.7 \%)$ & 0 & $2(1.2 \%)$ \\
\hline Unstable INR values & 0 & 0 & 0 \\
\hline Age $>65$ years & 45 (38.8\%) & $26(55.3 \%)$ & $71(43.6 \%)$ \\
\hline Blood thinner & $30(25.9 \%)$ & $17(36.2 \%)$ & $47(28.8 \%)$ \\
\hline Alcohol abuse & $16(13.8 \%)$ & 0 & $16(9.8 \%)$ \\
\hline Coagulopathy prior to ICU & $2(1.7 \%)$ & 0 & $2(1.2 \%)$ \\
\hline Indication for oral anticoagulation prior to ICU & $24(20.7 \%)$ & $9(19.1 \%)$ & $33(20.2 \%)$ \\
\hline Any oral anticoagulation ${ }^{\mathrm{a}}$ & $20(17.2 \%)$ & $10(21.3 \%)$ & $30(18.4 \%)$ \\
\hline Aspirin & $13(11.2 \%)$ & $9(19.1 \%)$ & $22(13.5 \%)$ \\
\hline Any platelet therapy & $16(13.8 \%)$ & $13(27.7 \%)$ & $29(17.8 \%)$ \\
\hline Any dual platelet therapy & $3(2.6 \%)$ & $3(6.4 \%)$ & $6(3.7 \%)$ \\
\hline \multicolumn{4}{|l|}{ aPTT target } \\
\hline$<40$ s (thromboprophylaxis) & $51(44.0 \%)$ & $14(29.8 \%)$ & $65(39.9 \%)$ \\
\hline $40-50 s$ & $19(16.4 \%)$ & $4(8.5 \%)$ & $23(14.1 \%)$ \\
\hline $50-70 s$ & $7(6.0 \%)$ & $8(17.0 \%)$ & $15(9.2 \%)$ \\
\hline 60-80s (therapeutic) & 39 (33.6\%) & $21(44.7 \%)$ & $60(36.8 \%)$ \\
\hline Unfractionated heparin & $115(99.1 \%)$ & $43(91.5 \%)$ & $158(96.9 \%)$ \\
\hline Argatroban & $7(6.0 \%)$ & $12(25.5 \%)$ & $19(11.7 \%)$ \\
\hline
\end{tabular}

Risk factors for bleeding are displayed for all patients, in non-COVID-19-ARDS and patients with COVID19-ARDS. Data are $\mathrm{n}(\%)$ or mean with standard deviation and range. ${ }^{a}$ One patient received fibrinolysis and was not included in this group. ${ }^{b} 158 / 163$ UFH, 18 were switched to argatroban, 1 patient directly received argatroban. 4 patients received only LMWH. If values are significantly different between nonCOVID-19 and COVID-19-patients, they are marked bold: any platelet therapy: $p=0.023$, alcohol abuse: $p=0.007$, UFH: $p=0.01$, argatrobran: $p<0.001$

Table 3: characteristics of intracerebral hemorrhage 


\begin{tabular}{|c|c|c|c|}
\hline & non-COVID-19 & COVID-19 & all patients \\
\hline Number of patients & $116(71.2 \%)$ & $47(28.8 \%)$ & $163(100 \%)$ \\
\hline Cerebral imaging ${ }^{\mathrm{a}}$ performed & $70(60.3 \%)$ & $26(55.3 \%)$ & $96(58.3 \%)$ \\
\hline Intracerebral hemorrhage (ICH) detected & $13(11.2 \%)$ & $9(19.1 \%)$ & $22(13.5 \%)$ \\
\hline Fatal intracerebral hemorrhage & $3(2.5 \%)$ & $3(6.3 \%)$ & $6(3.7 \%)$ \\
\hline \multicolumn{4}{|l|}{ Characteristics of intracerebral hemorrhage } \\
\hline Typical & $1(7.7 \%)$ & 0 & $1(4.5 \%)$ \\
\hline Atypical & $7(61.5 \%)$ & $2(22.2 \%)$ & $9(40.9 \%)$ \\
\hline Multiple & 0 & 4 (44.4\%) & $4(18.2 \%)$ \\
\hline $\mathrm{SAB}$ & $3(23.1 \%)$ & $2(22.2 \%)$ & $5(22.7 \%)$ \\
\hline $\mathrm{SDH} / \mathrm{EDH}$ & $2(15.4 \%)$ & $1(11.1 \%)$ & $3(13.6 \%)$ \\
\hline Intraventricular hemorrhage & $4(30.7 \%)$ & $2(22.2 \%)$ & $6(27.3 \%)$ \\
\hline RASS on day of hemorrhage & $-3.4 \pm 1.6(-5-0)$ & $-3.1 \pm 2.2(-5-0)$ & $-3.2 \pm 1.8(-5-0)$ \\
\hline Length of stay until hemorrhage & $15.5 \pm 19.6(0-63)$ & $20.4 \pm 14.8(2-47)$ & $17.6 \pm 17.6(0-63)$ \\
\hline Hemorrhage during $\mathrm{IMV}^{\mathrm{b}}$ & $12(92.3 \%)$ & $7(77.8 \%)$ & $19(86.4 \%)$ \\
\hline Hemorrhage during RRT & $5(38.5 \%)$ & $3(33.3 \%)$ & $8(36.4 \%)$ \\
\hline Hemorrhage during ECMO & $9(69.2 \%)$ & $4(44.4 \%)^{\mathrm{C}}$ & $13(50.0 \%)$ \\
\hline HAS-BLED Score $48 \mathrm{~h}$ prior to hemorrhage & $2.3 \pm 1.3(0-4)$ & $2.3 \pm 1.4(0-4)$ & $2.3 \pm 1.3(0-4)$ \\
\hline Blood pressure excession $48 \mathrm{~h}$ prior to hemorrhage & $3(23.1 \%)$ & $2(22.2 \%)$ & $5(27.3 \%)$ \\
\hline aPTT excess $48 \mathrm{~h}$ prior to hemorrhage & $3(23.1 \%)$ & $1(11.1 \%)$ & $4(18.0 \%)$ \\
\hline
\end{tabular}

Characteristics of intracerebral hemorrhage are displayed for all patients, in non-COVID-19-ARDS and patients with COVID-19-ARDS. Data are $\mathrm{n}(\%)$ or mean with standard deviation and range. ${ }^{\text {a }} 93$ cerebral computed tomographies (cCT) only, 2 cerebral magnet resonance tomograophies (cMR) only, $6 \mathrm{cCT}$ and CMR. ${ }^{b}$ no ICH under non-invasive ventilation or nasal high flow. ${ }^{c}$ in one patient $\mathrm{ICH}$ was detected after ECMO weaning but associated with ECMO-therapy and included in this group. If values are significantly different between non-COVID-19 and COVID-19-patients, they are marked bold: multiple ICH $p=0.0172$.

\section{Figures}




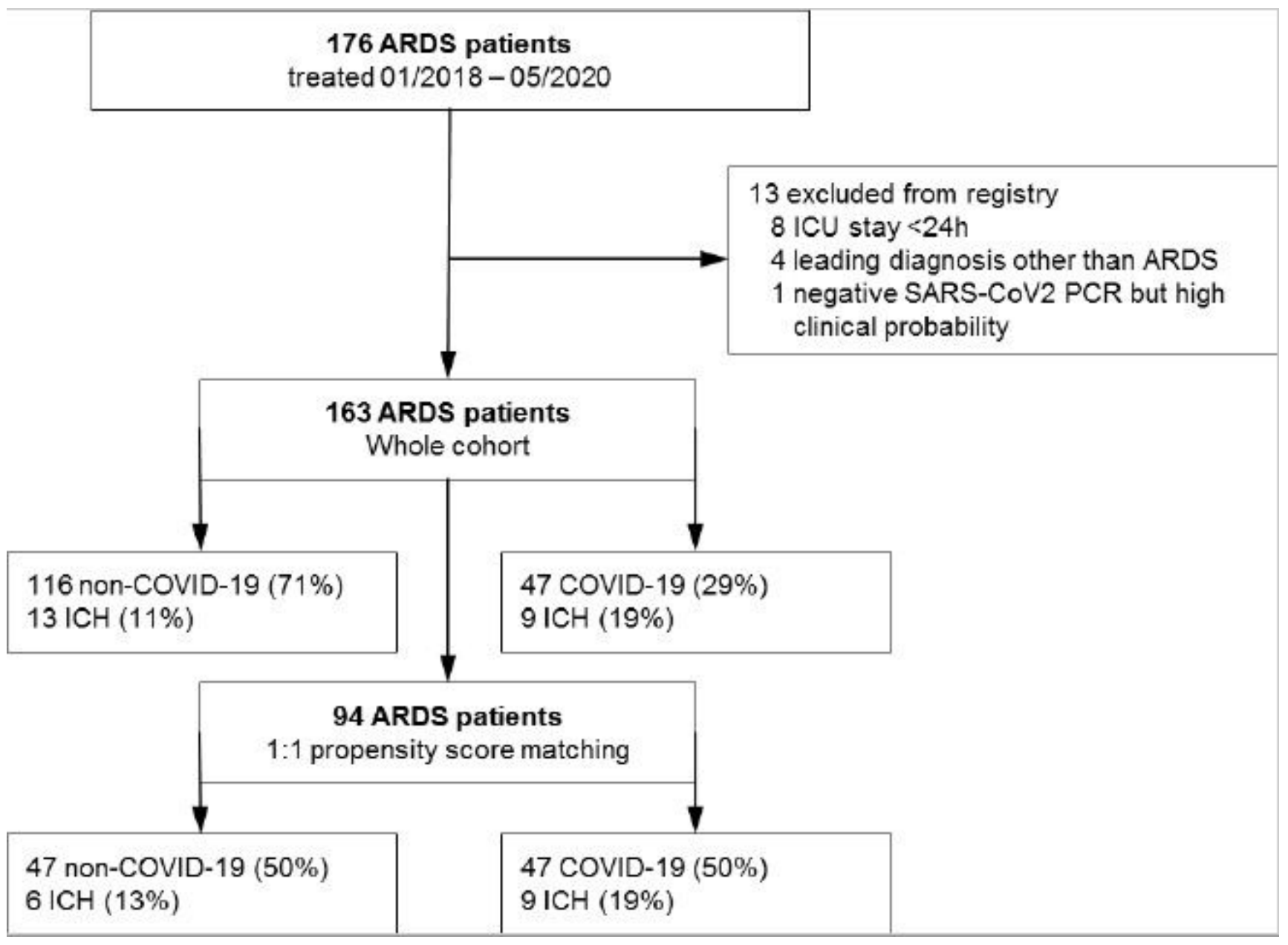

\section{Figure 1}

Patient flow chart Flow chart of patients in registry. Abbreviations: ARDS acute respiratory distress syndrome, ICH intracerebral hemorrhage 


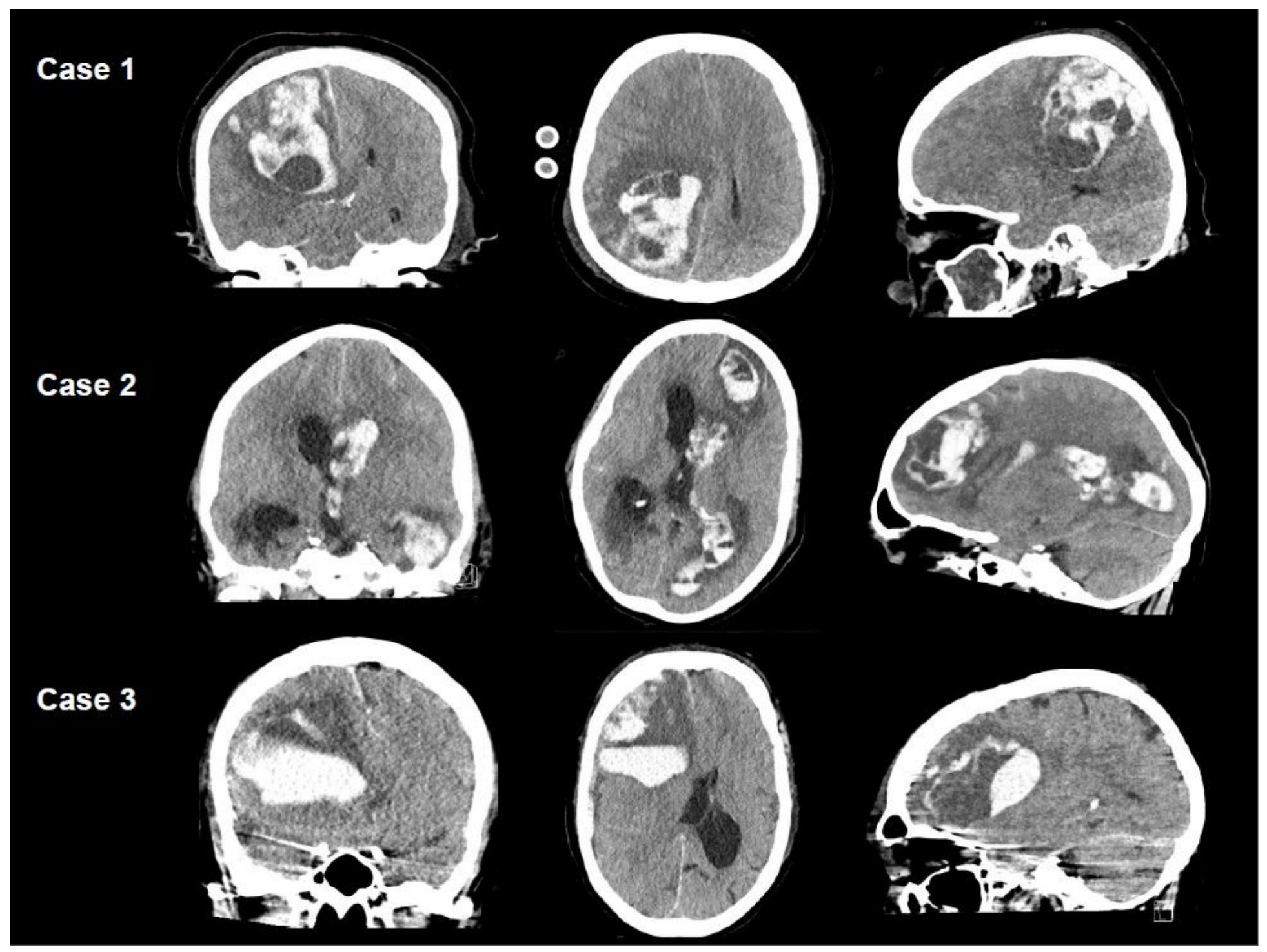

Figure 2

Computed tomographies of three COVID-19 intracerebral hemorrhages The computed tomographies show three cases of fatal intracerebral hemorrhages in COVID-19 patients with severe pulmonary failure. Case 1: 60-year old female obese health-care worker undergoing steroid therapy due to fibromyalgia. SARS-CoV2 pneumonia resulted in severe ARDS on ECMO and RRT. On day 19 of her clinical course and day 10 after ECMO implantation, she presented with anisocoric pupils. The intracerebral hemorrhage was judged fatal. Case 2: 49-year old otherwise healthy male with severe ARDS on ECMO after SARS-CoV2 transmission presumably at his family doctor's practice. On day 7 after hospitalization and on day 3 after ECMO implantation a fatal intraparenchymatoes hemorrhage occurred. Liquor remained negative in the virological testing according to SARS-CoV2. Case 3: 69-year old male with a coronary artery disease and atrial fibrillation with COVID-19 ARDS. On day 9, he presented with anisocoric pupils and the CCT revealed a right frontal cerebral bleeding. Evacuation of the bleeding was performed but the patient deceased. Neuropathology work-up showed a fresh parenchymal and subarachnoidal bleeding and reactive gliotic tissue. 


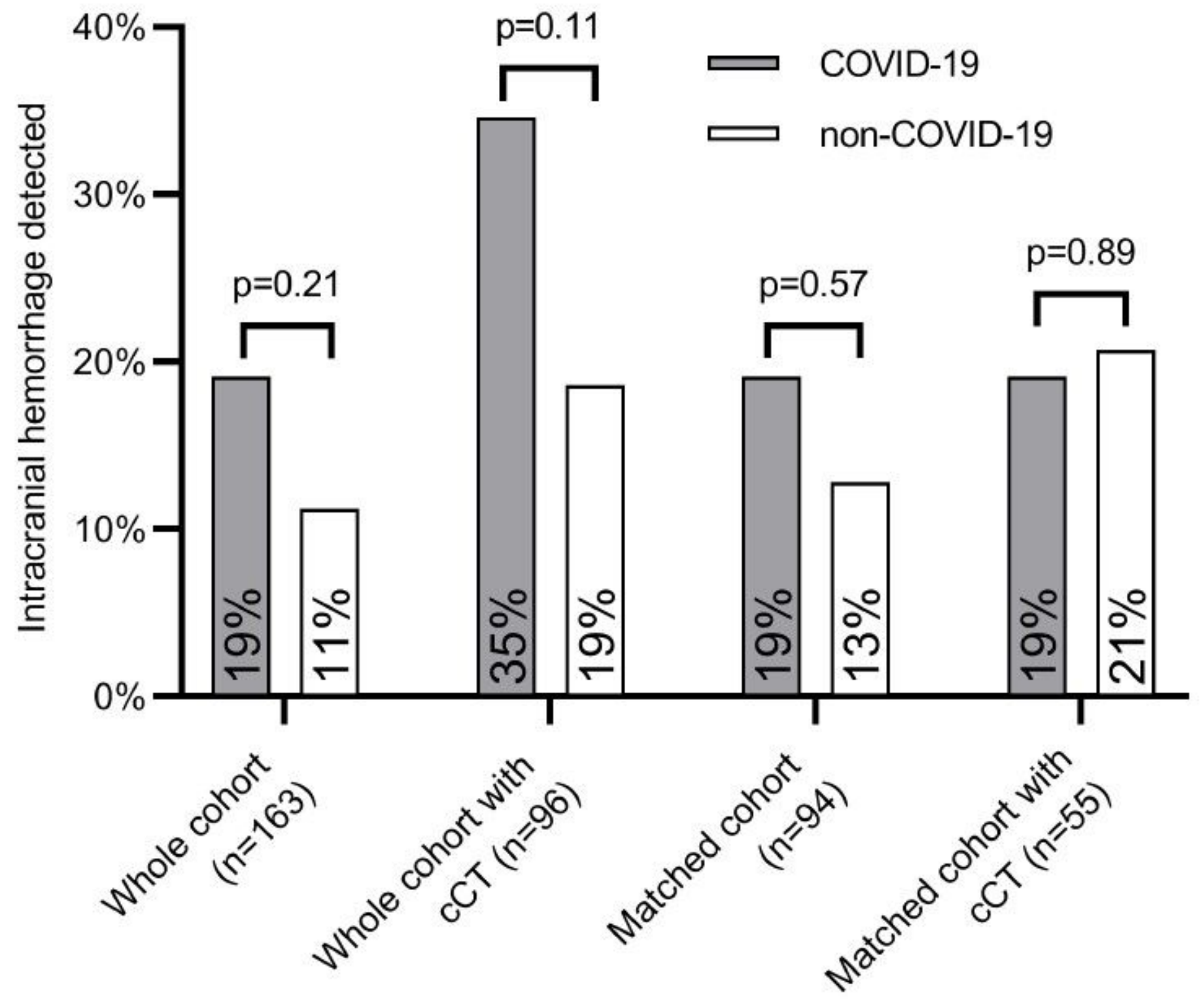

Figure 3

Intracerebral hemorrhage in acute respiratory distress syndrome There was no significant difference between patients with and without COVID-19 in respect to intracerebral hemorrhage as diagnosed by cerebral computed tomography (cCT).

\section{Supplementary Files}

This is a list of supplementary files associated with this preprint. Click to download.

- TableS1.png

- Tables2.png 
- Tables3.png

- Tables4.png

Page 18/18 